\title{
The Clinical Significance of FilmArray Respiratory Panel in Diagnosing Community-Acquired Pneumonia
}

\author{
Huanzhu Chen, ${ }^{1}$ Huilan Weng, ${ }^{2}$ Meirui Lin, ${ }^{2}$ Ping He, ${ }^{1}$ Yazhen Li, ${ }^{1}$ Qingdong Xie, ${ }^{1}$ \\ Changwen Ke, ${ }^{1}$ and Xiaoyang Jiao ${ }^{1}$ \\ ${ }^{1}$ Cell Biology and Genetics Department, Shantou University Medical College, Shantou 515041, China \\ ${ }^{2}$ First Affiliated Hospital of Shantou University Medical College, Shantou 515041, China \\ Correspondence should be addressed to Changwen Ke; kecw1965@aliyun.com and Xiaoyang Jiao; xyjiao@stu.edu.cn
}

Received 10 May 2017; Accepted 11 July 2017; Published 6 September 2017

Academic Editor: Graciela Russomando

Copyright (C) 2017 Huanzhu Chen et al. This is an open access article distributed under the Creative Commons Attribution License, which permits unrestricted use, distribution, and reproduction in any medium, provided the original work is properly cited.

Aim. FilmArray Respiratory Panel (FilmArray RP) test is an emerging diagnostic method in fast detecting multiple respiratory pathogens; the methodology and clinical significance of FilmArray RP in community-acquired pneumonia (CAP) diagnosis were evaluated in this study. Methods. Specimens from 74 patients with CAP were analyzed and compared using FilmArray RP, traditional multiple PCR assay, bacterial (or fungal) culture, and serological detection. Results. FilmArray RP and multiplex PCR showed 100\% coincidence rate in detecting coronaviruses 229E, OC43, HKU1, and NL63, human metapneumovirus, influenza A and B, and parainfluenza viruses (PIV1, PIV2, and PIV4). There were 15 viral specimens tested as disagreement positive results. FilmArray RP had higher detection rate in detecting dual viral and Mycoplasma pneumoniae infection. The positive bacteria (or fungi) were found in 25 specimens. Conclusions. This study demonstrated the capability of FilmArray RP for simultaneous detection of broadspectrum respiratory pathogens and potential use in facilitating better patient care.

\section{Introduction}

The community-acquired pneumonia (CAP) is the leading cause of morbidity and mortality worldwide. According to WHO estimates, 450 million cases of pneumonia are recorded each year, with about 4 million deaths from this illness $[1,2]$, with the highest incidence cases in children younger than 5 years old [3]. The mortality rate has declined greatly due to early and accurate detection of the etiological agents, together with the timely initiation of appropriate treatment [4].

Nowadays, the golden standard for CAP diagnosis is still based on the chest radiography; however, a broad range of chest radiographic changes could be induced by various agents, a single and dual bacterium/virus, or mixed pathogens coinfections. These alterations of chest radiography are only helpful in specific cases to confirm a microbial cause of pneumonia [4]. Current diagnostic methods identify a pathogen in only $30 \%-40 \%$ of CAP patients $[5,6]$, and the frequent lack of a microbiological diagnosis in CAP impairs pathogendirected antimicrobial therapy [7]. Polymerase chain reaction (PCR) technique has been shown to be reliable for diagnosing the pathogens, especially for those that are difficult to culture [4]. In addition, PCR method has high sensitivity and specificity in detecting multiple microorganisms and yields results faster than culture and serological methods [8]. Most importantly, the results of PCR are not affected by prior use of antibiotics. The findings indicate that the incidence of viral pneumonia in the past has been underestimated [4]. With multiple PCR assay, detection of multiple RNA or DNA targets in a single tube has the potential for rapid identification of complicated respiratory viral pathogens $[9$, 10]. The conventional techniques of microbial detection have high accuracy, but they are time- and labor-intensive, with limited range of detection and can be subjective, relying very much on technical expertise for the interpretation of cytopathic effect (CPE) in cell culture [11]. Direct fluorescent antibody assay (DFA) and immunochromatographic antigen 
testing, although rapid, have poor sensitivity for the detection of most viruses [12]. The serological methods also have low sensitivity. Therefore, an assay that is capable of rapid detection and accurate identification of multiple pathogens is desirable.

FilmArray Respiratory Panel (RP) is a multiplexed nucleic acid test for the simultaneous qualitative detection and identification of multiple respiratory virus, Bordetella pertussis, Chlamydophila pneumoniae, and Mycoplasma pneumoniae. Particularly, the whole process only takes about an hour; compared to multiple PCR, the FilmArray RP has provided fast results. As the FilmArray RP is emerging method, which is rarely employed in clinical specimen's detection, its clinical significance in diagnosing CAP is sparse. Therefore, in this study, we evaluated the capability of FilmArray RP for simultaneous identification of multiple pathogens; its microbial yield and clinical significance in CAP diagnosis were also evaluated.

\section{Materials and Methods}

2.1. Clinical Specimens. In all specimens of patients from the first and the second affiliated hospitals of Shantou University Medical College, CAP was confirmed by radiological diagnosis. The nasal cavity samples and serum were collected from patients between January and September, 2016.

2.2. Multiplex Real-Time PCR Assay. The specimens collected from nasal mucus were stored in swab storage solution (COPAN, Italy) for multiplex PCR and FilmArray RP assay. The nucleic acid was extracted by using beads viral DNA/RNA extraction kit (TIANLONG, China) and NP968 nucleic acid extraction instrument (TIANLONG, China). We tested 14 viruses, including influenza viruses types $A$ and $B$ (Flu A and Flu B), parainfluenza viruses 1 to 4 (PIV1-4), respiratory syncytial virus (RSV), coronaviruses 229E, OC43, HKU1, and NL63, human metapneumovirus (MPV), human rhinovirus (HRV), and adenovirus (ADV), were detected using a single-tube TaqMan ${ }^{\circledR}$ real-time reverse transcription PCR strategy (AgPath-ID ${ }^{\text {TM }}$ One-Step RT-PCR Kit Applied Biosystems, USA). PCR amplification was performed using a 7500 Real-Time PCR System (Applied Biosystems, USA), according to the manufacturer's instructions.

2.3. Bacterial (or Fungi) Detection. The nasal mucus specimens were cultured according to China national standard protocols to detect respiratory bacterial (or fungi). Colony identification was undertaken using a VITEK 2 Compact system (Biomérieux, France). Nine bacteria were measured, including Acinetobacter baumannii, Enterobacter aerogenes, Escherichia coli, Klebsiella ornithinolytica, Klebsiella pneumoniae, Pseudomonas aeruginosa, Staphylococcus aureus, Staphylococcus haemolyticus, and Bordetella pertussis. One fungus (yeast) was also detected.

2.4. Mycoplasma pneumoniae and Chlamydia pneumoniae Detection. The patient's serum was collected and placed in $-80^{\circ} \mathrm{C}$. Mycoplasma pneumoniae and Chlamydia pneumoniae antibody (IgM) were detected by commercial kit (KANGHUA, China), in accordance with the instructions.

2.5. The FilmArray RP Assay. We used the BioFire ${ }^{\mathrm{TM}}$ Diagnostics Respiratory Panel and FilmArray ${ }^{\mathrm{TM}}$ multiplex PCR system (Biomérieux, USA) to detect the same 14 viruses and three other pathogens (Bordetella pertussis, Chlamydophila pneumoniae, and Mycoplasma pneumoniae) according to the package insert.

2.6. Statistical Analyses. Data were analyzed in SPSS 19.0. The agreement between assays was measured using the kappa statistic. The sensitivity and specificity were also compared for both tests.

\section{Results}

3.1. Patients' Biographic and Clinical Features. Patients were enrolled between January and September, 2016. This study was comprised of both adult $(n=37)$ and pediatric $(n=37)$ patients with female/male ratio of $0.68: 1$. Patients in this study could have received treatment with antibiotics (cephalosporins, astaxanthin, and sulbactam, resp.) and antiviral drugs (oseltamivir and ribavirin), in combination or alone. Four cases of patients had the other lung diseases, while 32 cases of patients had system diseases. Cough, fever, and shortness of breath or dyspnea were the most frequently symptoms observed in these patients. General characters are presented in Table 1.

3.2. Virus Detection in CAP Patients. The most prevalent were the $\operatorname{RSV}(15 / 74,20.3 \%)$ and the HRV $(9 / 74,12.2 \%)$. Influenza viruses $(A+B)$ were detected in $10(10 / 74,13.5 \%)$ samples. Coronaviruses (OC43, HKU1, and NL63) were detected in 9 $(9 / 74,12.2 \%)$ samples, PIV3 in $5(5 / 74,6.8 \%)$ samples, ADV in $3(3 / 74,4.1 \%)$ samples, and MPV in $2(2 / 74,2.7 \%)$ samples (Table 2).

3.3. Correlation between FilmArray RP and Conventional Multiplex PCR Assay. For viruses 229E, OC43, HKU1, NL63, MPV, Flu A, Flu B, PIV1, PIV2, and PIV4, two methods had very good agreement with coincidence rate of $100 \%$. Disagreement was found in 15 specimens, positive results were tested by only one of the two assays (FilmArray RP: 12 cases, multiple PCR: 3 cases). FilmArray RP positive/PCR negative were seen in 3 cases of ADV, 6 cases of HRV, and 3 cases of RSV, while multiple PCR positive/FilmArray RP negative were observed in 1 case of PIV 3 and 2 cases of RSV (Table 2).

3.4. Sensitivity and Specificity of FilmArray RP in Detecting Virus. In the study, the FilmArray RP detected 50 positive viruses (ADV, 3; OC43, 7; HKU1, 1; NL63, 1; MPV, 2; HRV, 9; Flu A, 8; Flu B, 2; PIV3, 4; and RSV, 13, resp.). A total of $50(100 \%)$ of the 50 pathogens were confirmed by real-time PCR; the comparison of FilmArray RP and multiple PCR for OC43, HKU1, NL63, MPV, Flu A, Flu B, PIV3, and RSV 
TABLE 1: Patients' general characteristics.

\begin{tabular}{|c|c|}
\hline & Cases \\
\hline Total & 74 \\
\hline \multicolumn{2}{|l|}{ Age } \\
\hline $0-14$ & 37 \\
\hline$\geq 15$ & 37 \\
\hline \multicolumn{2}{|l|}{ Gender } \\
\hline Male & 44 \\
\hline Female & 30 \\
\hline \multicolumn{2}{|l|}{ Pathogen infection } \\
\hline Single virus & 26 \\
\hline Single bacterial (or fungi) & 10 \\
\hline Virus + bacterial (or fungi) & 14 \\
\hline Single Mycoplasma pneumoniae & 5 \\
\hline Virus + Mycoplasma pneumoniae & 1 \\
\hline Unidentified & 18 \\
\hline \multicolumn{2}{|l|}{ Treatment } \\
\hline Antibiotic & 45 \\
\hline Antiviral & 7 \\
\hline Antiviral + antibiotic & 11 \\
\hline \multicolumn{2}{|l|}{ Other lung diseases } \\
\hline COPD & 1 \\
\hline Tuberculosis & 1 \\
\hline Asthma & 2 \\
\hline \multicolumn{2}{|l|}{ Systemic diseases } \\
\hline Diabetes & 4 \\
\hline High blood pressure & 14 \\
\hline Heart disease & 3 \\
\hline Tumor & 1 \\
\hline Hepatitis & 6 \\
\hline Epilepsy & 4 \\
\hline \multicolumn{2}{|l|}{ Severity } \\
\hline Mild-moderate & 47 \\
\hline Severity & 27 \\
\hline \multicolumn{2}{|l|}{ Clinical symptom } \\
\hline Cough & 56 \\
\hline Pharyngeal discomfort & 4 \\
\hline Snivel & 10 \\
\hline Fever & 42 \\
\hline Shortness of breath or dyspnea & 25 \\
\hline \multicolumn{2}{|l|}{ Prognosis after healing } \\
\hline Death & 3 \\
\hline Alive & 71 \\
\hline
\end{tabular}

COPD, chronic obstructive pulmonary disease.

showed satisfactory agreement (kappa $>0.7$ ) for all positive viruses except ADV and HRV (kappa $=0$; kappa $<0.6$ ). In total, FilmArray RP and multiple PCR had significantly high sensitivities and specificities for the detection of respiratory viruses, but compared to the multiple PCR, the sensitivity of FilmArray RP for PIV3 and RSV was different, with sensitivity of $80 \%$ and $83.3 \%$, respectively (Table 2 ).
3.5. Dual Viral Infection Detected by FilmArray RP and Traditional Multiplex PCR Assay. Dual viral infections were detected in $12(12 / 74,16.2 \%)$ specimens (Table 3). Among them, 5 cases of dual viral infection were found in both methods, 6 cases were detected by FilmArray RP alone, and 1 case was detected by multiple PCR. In dual viral infection, majority was RSV combined with other respiratory viruses, a total of 9 cases, such as RSV + HRV positive (3 cases), RSV + ADV positive (2 cases), RSV + Flu A positive (1 case), RSV + HKU1 positive (1 case), RSV + NL63 positive (1 case), and RSV + PIV3 positive (1 case). The data indicated that FilmArray RP detected more viruses than multiple PCR, especially in dual viral infections. Intriguingly, the nondetected virus by PCR assay concentrated on RSV is accompanied by other respiratory virus.

3.6. Detection of Bacterial (or Fungi) or Mycoplasma pneumoniae Infections. Bacteria (or fungi) were detected by culture method in our study. The results showed that bacteria (or fungi) were positive in $25(25 / 74,33.8 \%)$ specimens. As FilmArray RP only detects Bordetella pertussis, it is hard to do method evaluation between two methods. In our study, 2 cases of Bordetella pertussis were detected by FilmArray RP. However, no Bordetella pertussis was isolated by culture method. The Mycoplasma pneumoniae found in $6(6 / 74,8.1 \%)$ specimens was only to be detected by FilmArray RP, while all serological tests to Mycoplasma pneumoniae were negative (Table 4).

\section{Discussion}

Microorganism measurement is an integral part of CAP patient management. Accurate and rapid etiological diagnosis helps prevent secondary infection, prevent the use of unnecessary antibiotics, facilitate more timely use of antiviral drugs, and shorten hospital stays [13]. In our study, even with bacterial (or fungal) culture, antibody IgM detection, multiple PCR, and FilmArray RP, there were 18/74 cases (24.32\%) of CAP that could not find the pathogens.

The multiplex real-time PCR can detect a broad spectrum of respiratory microorganisms promptly, which makes it possible for clinician to treat the patients with specific antimicrobial agents. However, the main limitations of PCR method include the handling of whole batch samples (at least 8 samples) for cost and labor saving, the different boxes to do extraction, the training of specialized biologist that may be the main reason of DNA/RNA extraction and amplification being easily contaminated, and results interpretation (the frequency of false positive or false negative results hinders the use of PCR in clinical practice). Thus, the PCR is not appropriate for instant point of care especially to some severe patients. Compared with multiple PCR, the measurement time of FilmArray RP needs only about 1.2 hours and there is no need to do the experiment in batch. The single test set makes it meet the requirement of clinician to decide whether the patients should be quarantined or not. For some virus, such as Flu A, rapid diagnosis may help prevent secondary 
TABLE 2: Comparison of positive and negative results in two methods in viral detection.

\begin{tabular}{|c|c|c|c|c|c|c|c|c|c|}
\hline \multirow{2}{*}{ Virus } & \multicolumn{4}{|c|}{ Number of specimens (+: positive; -: negative) } & \multirow{2}{*}{ Sensitivity (\%) } & \multirow{2}{*}{ Specificity (\%) } & \multirow{2}{*}{ PPV (\%) } & \multirow{2}{*}{ NPV (\%) } & \multirow{2}{*}{ Kappa } \\
\hline & PCR-/FA- & $\mathrm{PCR}+/ \mathrm{FA}+$ & $\mathrm{PCR}+/ \mathrm{FA}-$ & PCR-/FA+ & & & & & \\
\hline $\mathrm{ADV}$ & 71 & 0 & 0 & 3 & 0 & 95.9 & 0 & 100 & 0 \\
\hline 229E & 74 & 0 & 0 & 0 & 0 & 100 & 0 & 100 & 1 \\
\hline OC43 & 67 & 7 & 0 & 0 & 100 & 100 & 100 & 100 & 1 \\
\hline HKU1 & 73 & 1 & 0 & 0 & 100 & 100 & 100 & 100 & 1 \\
\hline NL63 & 73 & 1 & 0 & 0 & 100 & 100 & 100 & 100 & 1 \\
\hline MPV & 72 & 2 & 0 & 0 & 100 & 100 & 100 & 100 & 1 \\
\hline HRV & 65 & 3 & 0 & 6 & 100 & 91.5 & 33.3 & 100 & 0.468 \\
\hline FluA & 66 & 8 & 0 & 0 & 100 & 100 & 100 & 100 & 1 \\
\hline FluB & 72 & 2 & 0 & 0 & 100 & 100 & 100 & 100 & 1 \\
\hline PIV1 & 74 & 0 & 0 & 0 & 0 & 100 & 0 & 100 & 1 \\
\hline PIV2 & 74 & 0 & 0 & 0 & 0 & 100 & 0 & 100 & 1 \\
\hline PIV3 & 69 & 4 & 1 & 0 & 80 & 100 & 100 & 98.6 & 0.882 \\
\hline PIV4 & 74 & 0 & 0 & 0 & 0 & 100 & 0 & 100 & 1 \\
\hline RSV & 59 & 10 & 2 & 3 & 83.3 & 95.2 & 76.9 & 96.7 & 0.752 \\
\hline
\end{tabular}

FA, FilmArray RP; PPV, positive predictive value; NPV, negative predictive value.

TABLE 3: The positive virus combination of FilmArray RP and multiplex real-time PCR in detecting dual viral infection.

\begin{tabular}{ccc}
\hline Multiplex PCR assay & FilmArray RP \\
\hline OC43 + HRV & OC43 + HRV \\
Flu A + MPV & Flu A + MPV \\
RSV + HKU1 & RSV + HKU1 \\
RSV + PIV3 & RSV + PIV3 \\
RSV + NL63 & RSV + NL63 \\
RSV & RSV + HRV \\
RSV & RSV + HRV \\
RSV & RSV + ADV \\
RSV & RSV + ADV \\
Flu A & $R S V+$ Flu A \\
OC43 & OC43 + HRV \\
$R S V+$ HRV & HRV \\
\hline
\end{tabular}

spread, beneficial for preventing virus nosocomial transmission. In children and adults, neuraminidase inhibitors reduce median time to resolution of symptoms by 0.5-2.5 days when administered within $48 \mathrm{~h}$ of onset of symptoms [14]. Early use of neuraminidase inhibitors reduces the development of complications such as pneumonia [15]. From these points, the FilmArray RP offers potential advantage in patient's treatment.

Due to undeveloped immune system, mixed infections with two or more respiratory viruses are common in children but are not easily detected by conventional methods; hence, the biological significance of dual infections currently is not well understood [16]. It will have more clinical significance to analyze the association between age and pathogens. In our study, FilmArray RP had higher sensitivity in detecting the dual viral infection than multiple PCR. Similarly, from previous study, RSV is the predominant virus inducing severe pneumonia in the population [17-19] and is found to be the
TABle 4: The positive pathogens of bacterial (or fungi) or Mycoplasma pneumoniae detection and FilmArray RP in specimens detection.

\begin{tabular}{lcc}
\hline $\begin{array}{l}\text { Bacterial (or fungi) or Mycoplasma } \\
\text { pneumoniae detection }\end{array}$ & $\begin{array}{c}\text { FilmArray RP } \\
\text { (-: not detected) }\end{array}$ & Cases \\
\hline Yeast & - & 6 \\
Negative & $\begin{array}{l}\text { Mycoplasma } \\
\text { pneumoniae }\end{array}$ & 6 \\
Staphylococcus aureus & - & 4 \\
Acinetobacter baumannii & - & 3 \\
Enterobacter aerogenes & - & 2 \\
Klebsiella pneumoniae & - & 2 \\
Pseudomonas aeruginosa & - & 2 \\
Escherichia coli & Bordetella & 1 \\
Escherichia coli & pertussis & 1 \\
Klebsiella ornithinolytica & - & 1 \\
Negative & - & 1 \\
Staphylococcus haemolyticus & Bordetella & 1 \\
\hline
\end{tabular}

most common cause of severe respiratory disease in infants [20]. RSA and Flu A continue to be the major causative agent in dual viral infection. In this study, the detection rate of rhinoviruses was higher than for other respiratory viruses, though its role in pneumonia is still questioned [21]. The prevalence of adenovirus-associated CAP is fairly low $(3 / 74$, $4.1 \%$ ), which is similar to the previous study, but this type of infection is important to recognize because it might induce severe and fatal necrotizing pneumonia (especially serotypes 3, 7, and 14) [22]. For this, PCR is substantially more sensitive for identification of adenovirus than antigen detection [23]. Due to the wide range of pathogens responsible for CAP, in moderate or severe infection, broad-spectrum antimicrobial 
cover should be initiated before deescalating to narrow spectrum pathogen-directed agents once a microbiological diagnosis has been made $[24,25]$. The ability to differentiate viral from bacterial pneumonia could have very important significance in patient's management, which may help clinicians treat patients more promptly and appropriately. In hospital, it is not uncommon of mix bacterial-viral coinfections. When bacterial culture identify positive, combining viral infection is frequently ignored by clinician. On the other hand, the decision to prescribe antibiotics may be influenced by the lag of diagnosis; especially in children, empirical antibiotic therapy is almost always initiated in these patients [26] because of their immunocompromised and concerns for severe disease. In previous study, Mycoplasma pneumoniae is a causative agent of community-acquired atypical pneumonia, and Chlamydophila pneumoniae is an obligate intracellular bacterium that causes acute respiratory infections and is a common cause of CAP [27]. We used serological method to detect Mycoplasma pneumoniae and Chlamydia pneumoniae rather than culture. To Mycoplasma pneumoniae and Chlamydia pneumonia detection, culture is highly specific but it is a complex process that has a long turnaround time, technically demanding, and offers limited sensitivity. Serology and PCR methods may provide a rapid diagnosis [28]. The limitations of serologic method include Chlamydia pneumoniae IgM or IgG reactivity might be caused by heterotypic antibodies. The test is evidently unable to discriminate between past and persistent infections [29]. Previously, weak positive results were frequently observed in Mycoplasma pneumoniae and Chlamydia pneumonia detection in our lab; the reason needs further clarification. Comparing the methods mentioned above, FilmArray RP has high specificity and sensitivity and could obtain result in a very short time.

For considering the cost of reagent, the FilmArray RP is more expensive than multiple PCRs; the reagent and consumable costs (in RMB) for the FilmArray RP and the multiple PCR were calculated to be $\$ 230$ and $\$ 40$ per patient, respectively. However, the FilmArray RP is essential for the administration of appropriate antiviral therapy, which dramatically shortens hands-on time (62.7 hours versus 1.2 hours); the reduction in average time to discontinuation of oseltamivir resulted in cost savings of approximately US $\$ 34.16$ per patient [30]. In addition, FilmArray RP results in overall decreased duration of antibiotic use of half a day, a shorter stay (a quarter of a day less) in the hospital following admission and reduced time spent in isolation (a third of a day less) for patients who were viral positive after implementation of the FilmArray RP $[12,31]$. Therefore, the clinical significance is far beyond its cost. Compared with multiple PCR, the FilmArray RP provides instant care testing results with a simplified enhanced workflow, compared with laboratory-developed PCR (LD-PCR) [32].

In the last decades, the emergence of severe acute respiratory syndrome (SARS), avian influenza A (H5N1) virus, and the 2009 pandemic influenza A (H1N1) virus has reemphasized the important role of respiratory viruses as causes of severe pneumonia [4]. Nowadays, newly discovered viruses, including bocavirus, PIV4, novel coronaviruses, and rhinoviruses, are increasingly being recognized as causes of respiratory illness [12], which lead to the extensive use of the FilmArray RP in detecting these novel viruses. One of limitations of the FilmArray RP is that it cannot be modified according to the needs of the clinicians and the laboratory, which could not add new viruses in the detection panel like the multiple PCR. Another limitation of the FilmArray RP is that it can only detect Bordetella pertussis, Chlamydophila pneumoniae, and Mycoplasma pneumoniae. Complicated bacterial (or fungal) cultures coexit in viral-bacterial (or fungi) infection; hence, from this point, bacterial (or fungal) cultures are essential for rational antibiotic selection and treatment success.

\section{Ethical Approval}

The study was approved by the Ethics Committee of Shantou University Medical College.

\section{Consent}

Written informed agreements were obtained from patients or their custodians.

\section{Conflicts of Interest}

The authors declare that the research was conducted in the absence of any commercial or financial relationships that could be construed as a potential conflict of interest.

\section{Authors' Contributions}

Huanzhu Chen, Huilan Weng, and Meirui Lin are equal contributors.

\section{Acknowledgments}

This study was supported by the Department of Education, Guangdong Government, under the Top-Tier University Development Scheme for Research and Control of Infectious Disease.

\section{References}

[1] I. Rudan, C. Boschi-Pinto, Z. Biloglav, K. Mulholland, and H. Campbell, "Epidemiology and etiology of childhood pneumonia," Bulletin of the World Health Organization, vol. 86, no. 5, pp. 408-416, 2008.

[2] WHO, "Revised global burden of disease 2002 estimates," http://www.who.int/healthinfo/global_burden_disease/estimates_regional_2002_revised/en/.

[3] R. E. Black, S. Cousens, H. L. Johnson et al., "Global, regional, and national causes of child mortality in 2008: a systematic analysis," The Lancet, vol. 375, no. 9730, pp. 1969-1987, 2010.

[4] O. Ruuskanen, E. Lahti, L. C. Jennings, and D. R. Murdoch, "Viral pneumonia," The Lancet, vol. 377, no. 9773, pp. 1264-1275, 2011.

[5] S. Jain, W. H. Self, R. G. Wunderink et al., "Community-acquired pneumonia requiring hospitalization among U.S. adults," 
The New England Journal of Medicine, vol. 373, no. 5, pp. 415427, 2015.

[6] D. M. Musher, I. L. Roig, G. Cazares, C. E. Stager, N. Logan, and H. Safar, "Can an etiologic agent be identified in adults who are hospitalized for community-acquired pneumonia: results of a one-year study," Journal of Infection, vol. 67, no. 1, pp. 11-18, 2013.

[7] N. J. Gadsby, C. D. Russell, M. P. Mchugh et al., "Comprehensive molecular testing for respiratory pathogens in communityacquired pneumonia," Clinical Infectious Diseases, vol. 62, no. 7, pp. 817-823, 2016.

[8] Y.-S. Chen, P.-Y. Liu, Y.-F. Huang et al., "Comparison of diagnostic tools with multiplex polymerase chain reaction for pediatric lower respiratory tract infection: a single center study," Journal of Microbiology, Immunology and Infection, vol. 46, no. 6, pp. 413-418, 2013.

[9] M. J. Loeffelholz, D. L. Pong, R. B. Pyles et al., "Comparison of the filmarray respiratory panel and prodesse real-time PCR assays for detection of respiratory pathogens," Journal of Clinical Microbiology, vol. 49, no. 12, pp. 4083-4088, 2011.

[10] S. K. Sanghavi, A. Bullotta, S. Husain, and C. R. Rinaldo, "Clinical evaluation of multiplex real-time PCR panels for rapid detection of respiratory viral infections," Journal of Medical Virology, vol. 84, no. 1, pp. 162-169, 2012.

[11] C. C. Ginocchio, "Detection of respiratory viruses using nonmolecular based methods," Journal of Clinical Virology, vol. 40, supplement 1, pp. S11-S14, 2007.

[12] N. E. Babady, P. Mead, J. Stiles et al., "Comparison of the Luminex xTAG RVP fast assay and the idaho technology FilmArray RP assay for detection of respiratory viruses in pediatric patients at a cancer hospital," Journal of Clinical Microbiology, vol. 50, no. 7, pp. 2282-2288, 2012.

[13] C. L. Byington, H. Castillo, K. Gerber et al., "The effect of rapid respiratory viral diagnostic testing on antibiotic use in a children's hospital," Archives of Pediatrics and Adolescent Medicine, vol. 156, no. 12, pp. 1230-1234, 2002.

[14] M. Shun-Shin, M. Thompson, C. Heneghan, R. Perera, A. Hamden, and D. Mant, "Neuraminidase inhibitors for treatment and prophylaxis of influenza in children: Systematic review and meta-analysis of randomised controlled trials," BMJ (Online), vol. 339, no. 7718, Article ID b3172, p. 449, 2009.

[15] H. Yu, Q. Liao, Y. Yuan et al., "Effectiveness of oseltamivir on disease progression and viral RNA shedding in patients with mild pandemic 2009 influenza A H1N1: opportunistic retrospective study of medical charts in China," BMJ (Online), vol. 341, no. 7775, Article ID c4779, p. 714, 2010.

[16] C. C. Ginocchio, F. Zhang, R. Manji et al., "Evaluation of multiple test methods for the detection of the novel 2009 influenza A (H1N1) during the New York City outbreak," Journal of Clinical Virology, vol. 45, no. 3, pp. 191-195, 2009.

[17] A. Legand, S. Briand, N. Shindo et al., "Addressing the public health burden of respiratory viruses: the Battle against Respiratory Viruses (BRaVe) Initiative," Future Virology, vol. 8, no. 10, pp. 953-968, 2013.

[18] J. A. Berkley, P. Munywoki, M. Ngama et al., "Viral etiology of severe pneumonia among Kenyan infants and children," JAMA - Journal of the American Medical Association, vol. 303, no. 20, pp. 2051-2057, 2010.

[19] H. Nair, D. J. Nokes, B. D. Gessner et al., "Global burden of acute lower respiratory infections due to respiratory syncytial virus in young children: a systematic review and meta-analysis," The Lancet, vol. 375, no. 9725, pp. 1545-1555, 2010.
[20] S. S. Mohapatra and S. Boyapalle, "Epidemiologic, experimental, and clinical links between respiratory syncytial virus infection and asthma," Clinical Microbiology Reviews, vol. 21, no. 3, pp. 495-504, 2008.

[21] T. Jartti, L. Jartti, V. Peltola, M. Waris, and O. Ruuskanen, "Identification of respiratory viruses in asymptomatic subjects: asymptomatic respiratory viral infections," Pediatric Infectious Disease Journal, vol. 27, no. 12, pp. 1103-1107, 2008.

[22] G. Carballal, C. Videla, A. Misirlian, P. V. Requeijo, and M. D. C. Aguilar, "Adenovirus type 7 associated with severe and fatal acute lower respiratory infections in Argentine children," BMC Pediatrics, vol. 2, no. 6, 2002.

[23] J. C. Arnold, K. K. Singh, A. S. Stephen, and M. Sawyer, "Undiagnosed respiratory viruses in children," Pediatrics, vol. 121, no. 3, pp. e631-e637, 2008.

[24] W. Lim S, S. Baudouin V, R. George C et al., "BTS guidelines for the management of community acquired pneumonia in adults: update 2009," Thorax, vol. 64, Supplement 3, no. 2009, pp. 1-55, 2009.

[25] L. A. Mandell, R. G. Wunderink, A. Anzueto et al., "Infectious diseases society of America/American thoracic society consensus guidelines on the management of community-acquired pneumonia in adults," Clinical Infectious Diseases, vol. 44, supplement 2, pp. S27-S72, 2007.

[26] K. Strålin, "Usefulness of aetiological tests for guiding antibiotic therapy in community-acquired pneumonia," International Journal of Antimicrobial Agents, vol. 31, no. 1, pp. 3-11, 2008.

[27] D. L. Hahn, A. A. Azenabor, W. L. Beatty, and G. I. Byrne, "Chlamydia pneumoniae as a respiratory pathogen.", Frontiers in bioscience : a journal and virtual library, vol. 7, pp. e66-76, 2002.

[28] W. R. Peeling, "Laboratory diagnosis of Chlamydia pneumoniae infections," The Journal of Infectious Diseasess, vol. 6, pp. 198203, 1995.

[29] E. Villegas, A. Sorlózano, and J. Gutiérrez, "Serological diagnosis of Chlamydia pneumoniae infection: limitations and perspectives," Journal of Medical Microbiology, vol. 59, no. 11, pp. 1267-1274, 2010.

[30] N. N. Pettit, S. Matushek, A. Charnot-Katsikas et al., "Comparison of turnaround time and time to oseltamivir discontinuation between two respiratory viral panel testing methodologies," Journal of Medical Microbiology, vol. 64, no. 3, pp. 312-313, 2015.

[31] B. B. Rogers, P. Shankar, R. C. Jerris et al., "Impact of a rapid respiratory panel test on patient outcomes," Archives of Pathology and Laboratory Medicine, vol. 139, no. 5, pp. 636-641, 2015.

[32] E. Song, H. Wang, D. Salamon, P. Jaggi, and A. Leber, "Performance characteristics of FilmArray respiratory panel v1.7 for detection of adenovirus in a large cohort of pediatric nasopharyngeal samples: One test may not fit all," Journal of Clinical Microbiology, vol. 54, no. 6, pp. 1479-1486, 2016. 


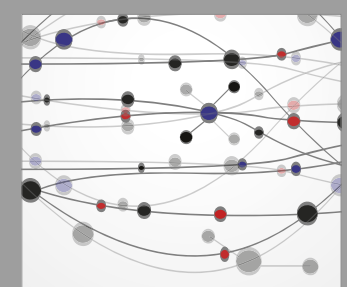

The Scientific World Journal
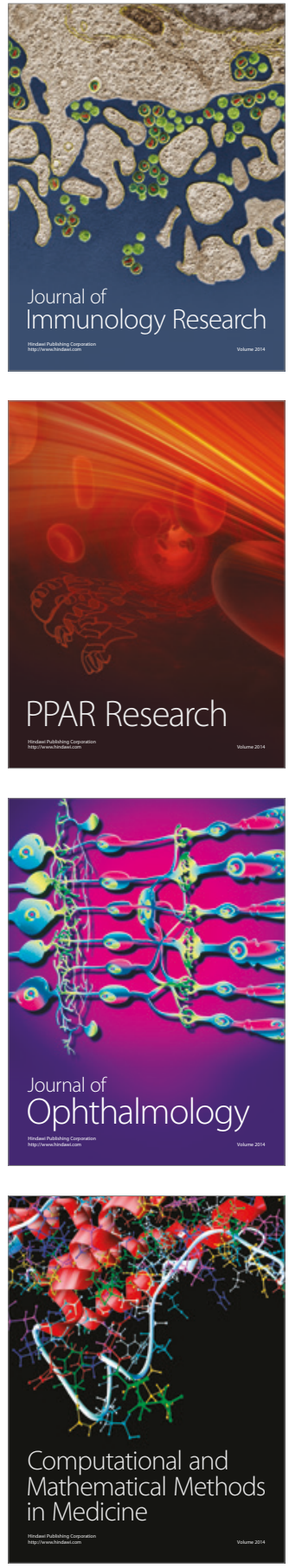

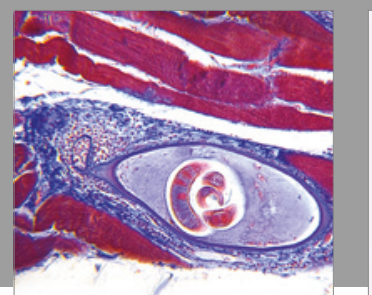

Gastroenterology Research and Practice
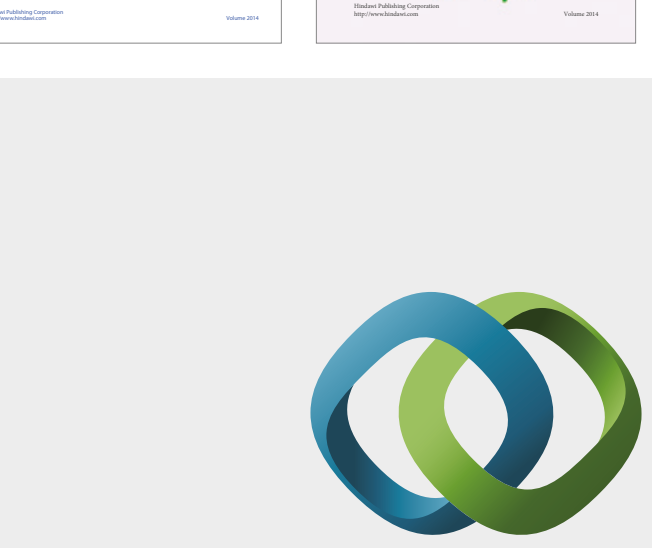

\section{Hindawi}

Submit your manuscripts at

https://www.hindawi.com
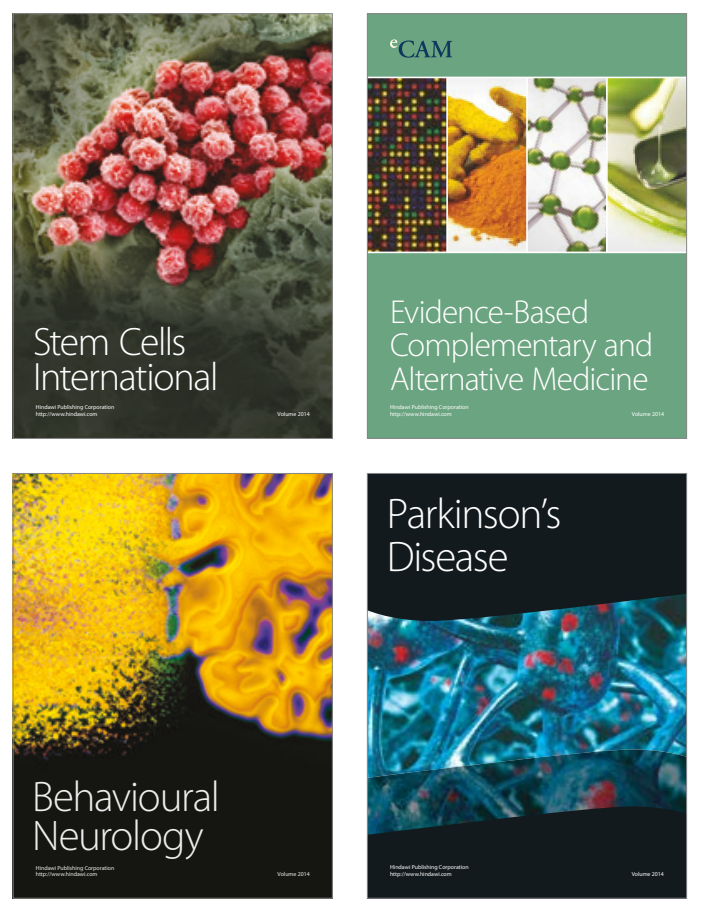
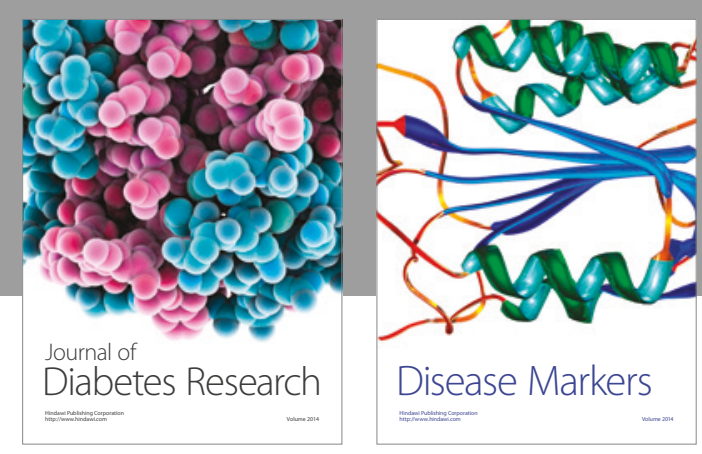

Disease Markers
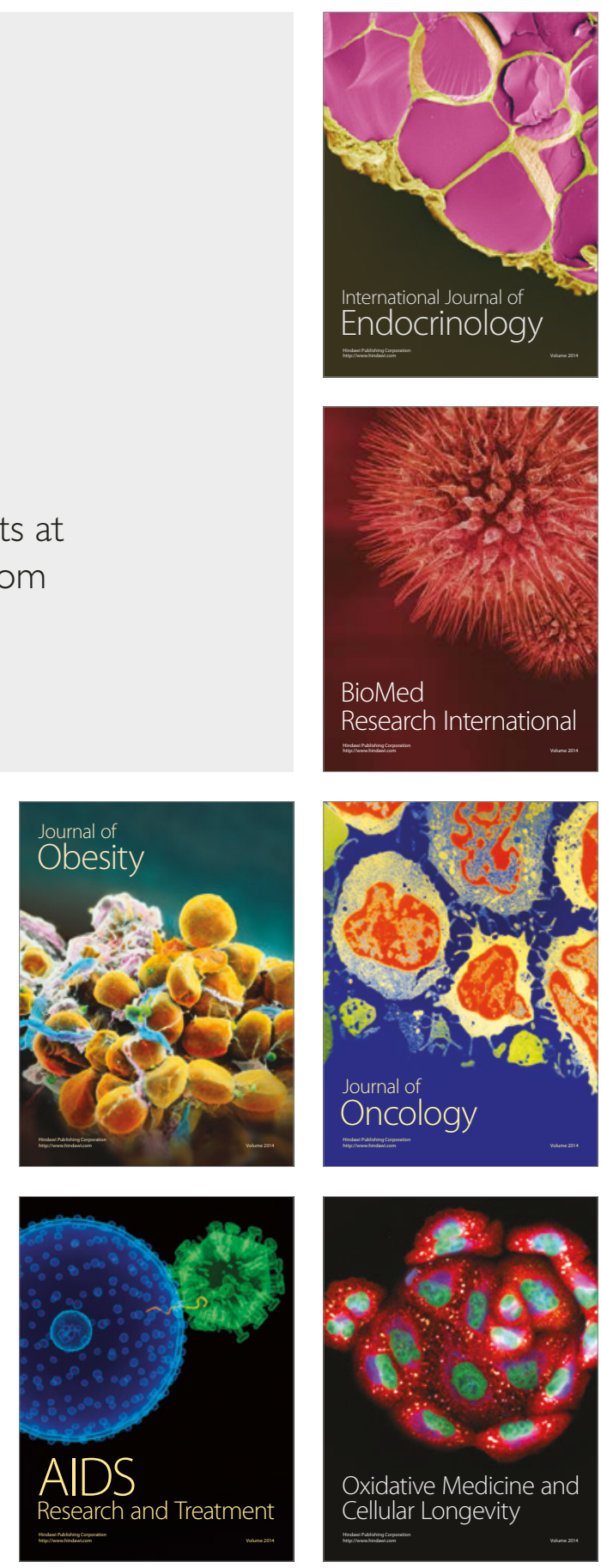